\title{
La vigencia de los mitos sobre la violencia contra las mujeres en la pareja
}

\section{The current acceptance of gender violence myths}

Fecha de recepción: 08/03/2016

Fecha de aceptación: 21/03/2016
Victoria Aurora Ferrer-Pérez

Catedrática de Universidad de Psicología Social de Género Departamento de Psicología, Universidad de las Islas Baleares

Lorena López-Prats

Colaboradora Departamento de Psicología, Universidad de las Islas Baleares

Esperanza Bosch-Fiol

Profesora Titular de Universidad de Psicología Básica Departamento de Psicología, Universidad de las Islas Baleares

Capilla Navarro-Guzmán

Grupo de Investigación de Estudios de Género. Universidad de las Islas Baleares Profesora Contratada Doctora de Psicología Social, Departamento de Psicología. Universidad de las Islas Baleares

\section{resumen/abstract:}

La violencia contra las mujeres en la pareja constituye un problema social y sanitario de primera magnitud, tal y como remarcan las principales autoridades sanitarias mundiales y nacionales. Sin embargo, y a pesar de ello, los mitos y las creencias erróneas sobre esta violencia aún se hallan fuertemente arraigados en muchos segmentos sociales. El presente estudio tiene como objetivo analizar la vigencia de algunos de estos mitos, particularmente, de aquellos relacionados con quienes cometen y padecen esta violencia, con sus causas y con la posible existencia de denuncias falsas. Para ello se estudió una muestra seleccionada por conveniencia y constituida por 423 personas de entre 17 y 81 años. Los resultados obtenidos apuntan que en torno a un tercio de las personas encuestadas tienen creencias erróneas sobre quienes padecen o cometen esta violencia o sobre la existencia de denuncias falsas, y, en general, estas diferencias prevalecen, independientemente del sexo, la edad o el nivel formativo. Estos resultados sugieren que es necesario seguir trabajando en la formación y la sensibilización social sobre esta violencia.

Intimate partner violence against women is a social and health problem of the first magnitude, as underline by global and national health authorities. However, despite this, the myths and misconceptions about this violence, its characteristics and its causes are still deeply rooted in many social segments. The present study aims to study the effect of some of these myths, particularly those related to perpetrators and victims of this violence, their causes and the possible existence of false complaints. For this purpose a sample, selected for convenience and consisting of 423 people aged 17 to 81 years, was studied. The results suggest that around a third of people surveyed have misconceptions about those who suffer or perpetrate such violence or the existence of false complaints, and, in general, these differences prevail, regardless of sex, age or study level. These results point out that it is necessary to continue working on training and social awareness about this violence.

\section{palabras clave/keywords:}

Vviolencia contra las mujeres; violencia de género; mitos

Violence against women; gender violence; myths

\section{Introducción}

En algunos trabajos anteriores (Bosch y Ferrer, 2002, 2012) se revisaron los principales mitos sobre la violencia contra las mujeres en la pareja (denominada violencia de género en el marco normativo español vigente), incluyendo su descripción, y evidencias disponibles 
y, en función de éstas, se realizó un análisis crítico sobre su posible veracidad. Como ya se apuntó en las conclusiones de dichos trabajos, el denominador común subyacente a muchos de estos mitos era, o bien situar el problema como algo alejado y relacionado con grupos o colectivos específicos (inmigrantes, parados, etc.), o con ciertas circunstancias biográficas o personales (familias desestructuradas, violentas, etc.), o bien tratar de resituar la responsabilidad de tal violencia, alejándola de los varones que la cometen.

A modo de recordatorio, cabe señalar que los mitos sobre la violencia de género son definidos conceptualmente como creencias estereotípicas sobre esta violencia, que son generalmente falsas pero sostenidas amplia y persistentemente, y que sirven para minimizar, negar o justificar la agresión a la pareja (Peters, 2008). Entre ellos estarían (Bosch y Ferrer, 2002; Peters, 2008) aquellos a los que se ha hecho referencia anteriormente, y que podemos considerar como "clásicos" (Bosch y Ferrer, 2012). Entre ellos se incluyen (San Martín y González, 2011): a) Los mitos sobre la marginalidad, que sitúan la violencia de género en el terreno de la excepcionalidad, manteniéndola como algo alejado del propio grupo y fruto de circunstancias excepcionales; b) Los mitos sobre los maltratadores, que ponen el acento en factores personales del agresor que le habrían llevado hasta la violencia y que, de un modo más o menos explícito, lo exoneran de culpa; o c) Los mitos sobre las mujeres maltratadas, que desplazan la carga de la culpa hacia ellas y las responsabilizan de lo que les sucede, bien sea porque algunas de sus características (de personalidad, de estatus, emocional,...) facilitarían la violencia, bien porque la consentirían o se mantendrían en situación de exposición a ella

A éstos, se sumarían los denominados neomitos (Lorente, 2009), esto es, nuevos mitos, evolucionados desde los tradicionales con un formato externo que, en apariencia, rompería con las posiciones tradicionales, ofreciendo un supuesto análisis desde la neutralidad y la defensa del bien común, pero que, en realidad, crean una referencia más compleja y/o mantienen y refuerzan las posiciones patriarcales más tradicionales en torno a esta violencia. Entre ellos estarían: a) El llamado Síndrome de Alienación Parental (SAP); b) La consideración de que las leyes criminalizan (considerando violencia de género) lo que son "conflictos normales" en las relaciones entre hombres y mujeres; c) La supuesta proliferación de denuncias falsas; o d) La consideración de los hombres como "verdaderas víctimas" del sistema (Lorente, 2009; Méndez, 2010). A todos ellos, habría que añadir, otros que, aunque no puedan ser considerados estrictamente como nuevos o recientes, irían en el mismo sentido que los neomitos. Se trata de aquellos a los que podríamos llamar "negacionistas" (Bosch y Ferrer, 2012)y que se centran en minimizar la importancia del problema, cuestionando la gravedad de esta violencia, e, incluso, su ocurrencia misma, considerando que la violencia de género es, en realidad, una exageración creada y utilizada por determinadas mujeres para obtener beneficios y/o perjudicar a los hombres, especialmente, en los litigios de separación y divorcio y por la custodia de los/as hijos/as.

Dada la proliferación de comentarios, noticias y reflexiones, más o menos contradictorias, sobre la vigencia o superación de estos mitos (tanto "antiguos" como "modernos"), se plantea este trabajo, cuyo objetivo es estudiar la vigencia de ciertos mitos sobre la violencia de género. Concretamente, este trabajo se centra de forma específica en analizar si persisten 
creencias erróneas sobre quienes cometen y padecen violencia de género, sobre la existencia de denuncias falsas y sobre las causas de esta violencia.

\section{Método}

\section{Participantes}

La muestra estuvo integrada por 423 personas de las que el 27.2\% $(n=115)$ eran varones, y el $72.8 \%(n=308)$ mujeres, de edades comprendidas entre los 17 y 81 años $(M=31.42$, $D T=14.89)$, predominando las personas de entre 17 y 23 años $(42.6 \%)$. El 9.2\% (n=39) tenían estudios primarios, el 21.5\% $(n=91)$ estudios secundarios, el 9.0\% $(n=38)$ estudios universitarios, y el 58.6\% ( $\mathrm{n}=248)$ eran estudiantes de nuestra universidad, concretamente, alumnado de Enfermería y Fisioterapia (41.1\%), de Psicología (28.6\%), de educación $(16.5 \%)$ y de otros estudios $(13.8 \%)$.

\section{Instrumentos.}

Para la realización de esta investigación se diseñó un cuestionario que incluyó las siguientes secciones:

a) Características sociodemográficas y de estudios. Se preguntó a los/as participantes por su sexo, edad, y nivel de estudios y, en el caso del alumnado universitario, por los estudios en curso.

b) Creencias sobre la violencia de género.Se realizaron preguntas abiertas en las que los/as participantes debían expresar, en formato libre, su opinión sobre la violencia de género y, particularmente, sobre: quienes la cometen, quienes la padecen, por qué ocurre, en qué edades es más frecuente, y si consideraban que se producen exageraciones o denuncias falsas en relación con esta violencia. Concretamente, se formularon preguntas como las siguientes: En relación a la violencia de género, cuál es tu opinión sobre: ¿Quiénes la cometen?, ¿Quiénes son las víctimas?, ¿En qué edades crees que es más frecuente esta violencia? En el caso de las causas por las que ocurre esta violencia, cada persona entrevistada podía señalar hasta tres causas, y éstas debían ser indicadas por orden de importancia.

\section{Procedimiento.}

La muestra estudiada fue seleccionada mediante un muestreo no probabilístico en el que se combinaron dos estrategias: Por una parte, se realizó un muestreo casual o incidental, seleccionando intencionadamente a los/as participantes de entre los/as estudiantes de las diferentes titulaciones de nuestra universidad. Por otra parte, se realizó un muestreo de bola de nieve, de modo que, a partir de los/as estudiantes encuestados, se accedió a personas de su entorno (familiares, amigos/as, etc.) de otras edades y niveles de estudios. De este modo fue posible obtener una muestra más amplia y diversa en cuanto a sus características, y, por tanto, más cercana a la composición real de la población general de nuestro entorno.

A las personas integrantes de la muestra se les entregó el cuestionario con las oportunas instrucciones para su cumplimentación y devolución. Todas las personas participantes fueron adecuadamente informadas del carácter voluntario y anónimo de su colaboración en el 
estudio, y de los objetivos del mismo, y aceptaron voluntariamente participar sin recibir ninguna compensación a cambio. A lo largo de todo el proceso, se respetaron en todo momento los preceptos de anonimato, confidencialidad y éticos de la investigación, comúnmente aceptados y establecidos en nuestra disciplina.

Tras la recepción de los cuestionarios, se procedió a categorizar las respuestas obtenidas. Para ello, se realizó una lectura comprensiva e individual de un número significativo de cuestionarios por parte de las personas integrantes del equipo investigador, y cada una de ellas elaboró una primera codificación de las mismas. Posteriormente, se revisó el acuerdo interjueces para las categorías obtenidas, conservando aquellas en las que había acuerdo, y reformulando o, en su caso, eliminando aquellas para las que había discrepancias. Una vez diseñado el sistema de categorización, se procedió a codificar la información para el conjunto de los cuestionarios recogidos.

\section{Diseño}

Este estudio se realizó a partir de un diseño seccional descriptivo, esto es, en el que la descripción se hace en un único momento temporal.

\section{Análisis de datos}

Para el análisis de la información sociodemográfica se ha realizado un análisis descriptivo con frecuencias y porcentajes. La comparación de grupos se realiza a partir de tablas de contingencia, el estadístico Chi cuadrado de Pearson, y el Coeficiente de contingencia (Cc) para determinar la existencia de asociación significativa entre variables, y, en su caso, la magnitud de tal asociación en función del sexo, la edad o el nivel de estudios. En todos lo casos, se estableció un valor $\mathrm{p}<0.05$ para determinar la significación estadística de los resultados. Para realizar estos análisis se utilizaron los programas estadísticos del paquete SPSS (versión 21 para Macintosh).

\section{Resultados}

Respecto a las creencias generales de la muestra sobre violencia de género (Tabla 1), 1lama la atención que tan solo un tercio tenga una opinión que se ajusta al marco normativo vigente, esto es, consideran que son los hombres quienes cometen la violencia de género y las mujeres quienes la padecen (33.1\% y $33.8 \%)$. Si bien es cierto que, tanto en un caso como en otro, este porcentaje se amplía cuando se incorporan ciertos matices (la cometen / la padecen personas de ambos sexos, pero mayoritariamente hombres /mujeres); o la cometen / la padecen hombres / mujeres con determinadas características), alcanzando hasta un $51.3 \%$ en el caso de señalar que la cometen varones, y un $46.6 \%$ en el caso de señalar que la padecen mujeres. Un tercio de las personas encuestadas (37.8\%) considera que no hay edad para la violencia de género, mientras casi otro tercio (31.7\%) considera que se trata de un problema más frecuente en la adultez que en otras etapas de la vida. Por otra parte, tan sólo una minoría (5.2\%) considera que se exagera la violencia de género, pero 3 de cada 10 personas encuestadas consideran que se ponen muchas denuncias falsas, especialmente, para obtener beneficios (como la custodia de los/as hijos/as o la vivienda) y por venganza (37.8\% y $37.0 \%$ de quienes consideran que efectivamente se ponen muchas denuncias falsas, respectivamente).

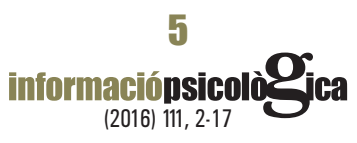


Tabla 1.- Creencias sobre la violencia de género.

\begin{tabular}{|c|c|c|}
\hline & & $\mathrm{n}(\%)$ \\
\hline \multirow{7}{*}{$\begin{array}{l}\text { Quiénes comenten } \\
\text { esta violencia }\end{array}$} & Hombres & $140(33.1)$ \\
\hline & $\begin{array}{l}\text { Personas de ambos sexos, pero mayoritariamente } \\
\text { hombres }\end{array}$ & $52(12.3)$ \\
\hline & Hombres con determinadas características & $25(5.9)$ \\
\hline & Personas de ambos sexos & $78(18.4)$ \\
\hline & Personas con determinadas características & $96(22.7)$ \\
\hline & $\begin{array}{l}\text { Depende de la violencia } \\
\text { (hombres-física, mujeres-psicológica) }\end{array}$ & $7(1.7)$ \\
\hline & $\mathrm{Ns} / \mathrm{Nc}$ & $25(5.9)$ \\
\hline \multirow{9}{*}{$\begin{array}{l}\text { Quiénes son } \\
\text { víctimas de esta } \\
\text { violencia }\end{array}$} & Mujeres & $143(33.8)$ \\
\hline & $\begin{array}{l}\text { Personas de ambos sexos, } \\
\text { pero mayoritariamente mujeres }\end{array}$ & $40(9.5)$ \\
\hline & Mujeres con determinadas características & $14(3.3)$ \\
\hline & Personas de ambos sexos & $92(21.7)$ \\
\hline & Personas con determinadas características & $70(16.5)$ \\
\hline & $\begin{array}{l}\text { Depende de la violencia } \\
\text { (hombres-física, mujeres-psicológica) }\end{array}$ & $2(0.5)$ \\
\hline & Pareja e hijos & $10(2.4)$ \\
\hline & Mujeres, niños y/o ancianos & $17(4.0)$ \\
\hline & $\mathrm{Ns} / \mathrm{Nc}$ & $35(8.3)$ \\
\hline \multirow{5}{*}{$\begin{array}{l}\text { En qué edad } \\
\text { crees que es más } \\
\text { frecuenteesta } \\
\text { violencia }\end{array}$} & No hay edad & $160(37.8)$ \\
\hline & Adolescencia-Juventud & $48(11.3)$ \\
\hline & Adultez & $134(31.7)$ \\
\hline & Juventud+Adultez & $61(14.4)$ \\
\hline & $\mathrm{Ns} / \mathrm{Nc}$ & $20(4.8)$ \\
\hline \multirow{2}{*}{$\begin{array}{l}\text { Crees que se } \\
\text { exagera el problema }\end{array}$} & $\mathrm{Si}$ & $22(5.2)$ \\
\hline & No & $401(94.8)$ \\
\hline \multirow{2}{*}{$\begin{array}{l}\text { Crees que se ponen } \\
\text { muchas denuncias } \\
\text { falsas }\end{array}$} & $\mathrm{Si}$ & $127(30.0)$ \\
\hline & No & $296(70.0)$ \\
\hline \multirow[t]{4}{*}{$\begin{array}{l}\text { En caso afirmativo, } \\
\text { por qué }\end{array}$} & $\begin{array}{l}\text { Obtener beneficios } \\
\text { (custodia, dinero, vivienda...) }\end{array}$ & $48(37.8)$ \\
\hline & Venganza & $47(37.0)$ \\
\hline & Otros & $31(24.4)$ \\
\hline & $\mathrm{Ns} / \mathrm{Nc}$ & $1(0.8)$ \\
\hline
\end{tabular}


Tabla 2.- Motivos por los que ocurre esta violencia según las personas encuestadas, por orden de importancia para las personas entrevistadas.

\begin{tabular}{|lcccc|}
\hline Motivo & Motivo 1 & Motivo 2 & Motivo 3 & Total \\
& $\mathrm{n}(\%)$ & $\mathrm{n}(\%)$ & $\mathrm{n}(\%)$ & $\mathrm{n}(\%)$ \\
\hline Sentimientos de superioridad & 61 & 50 & 47 & 158 \\
& $(14.4)$ & $(11.8)$ & $(11.1)$ & $(37.4)$ \\
Celos/envidia & 52 & 56 & 27 & 135 \\
& $(12.3)$ & $(13.2)$ & $(6.4)$ & $(31.9)$ \\
Problemas de pareja & 41 & 34 & 35 & 110 \\
& $(9.7)$ & $(8.0)$ & $(8.3)$ & $(26.0)$ \\
Machismo & 43 & 25 & 13 & 81 \\
& $(10.2)$ & $(5.9)$ & $(3.1)$ & $(19.1)$ \\
Alcoholismo/drogas & 24 & 30 & 27 & 81 \\
& $(5.7)$ & $(7.1)$ & $(6.4)$ & $(19.1)$ \\
Sociedad/cultura & 29 & 25 & 25 & 79 \\
& $(6.9)$ & $(5.9)$ & $(5.9)$ & $(18.7)$ \\
Problemas psicológicos/etc & 28 & 24 & 27 & 79 \\
& $(6.6)$ & $(5.7)$ & $(6.4)$ & $(18.7)$ \\
Carencias educativas & 40 & 19 & 16 & 75 \\
Falta de control emocional/persona & $(9.5)$ & $(4.5)$ & $(3.8)$ & $(17.7)$ \\
violenta & 16 & 24 & 26 & 66 \\
Antecedentes familiares de maltrato o & $(3.8)$ & $(5.7)$ & $(6.1)$ & $(15.6)$ \\
normas familiares & 26 & 20 & 17 & 63 \\
& $(6.1)$ & $(4.7)$ & $(4.0)$ & $(14.9)$ \\
Sentimientos de inferioridad & 17 & 23 & 17 & 57 \\
Emociones negativas (miedo, ira, & $(4.0)$ & $(5.4)$ & $(4.0)$ & $(13.5)$ \\
frustración, rabia, etc.) & 7 & 14 & 28 & 49 \\
Situación económica & $(1.7)$ & $(3.3)$ & $(6.6)$ & $(11.6)$ \\
& 6 & 18 & 13 & 37 \\
Desigualdad & $(1.4)$ & $(4.2)$ & $(3.1)$ & $(8.7)$ \\
Características de la víctima & 14 & 10 & 10 & 34 \\
Experiencias negativas o traumáticas & $(3.3)$ & $(2.4)$ & $(2.4)$ & $(8.0)$ \\
& 1 & 13 & 8 & 22 \\
& $(0.2)$ & $(3.1)$ & $(1.9)$ & $(5.2)$ \\
& 5 & 5 & 6 & 16 \\
& & & & \\
& $(1.2)$ & $(1.2)$ & $(1.4)$ & $(3.8)$
\end{tabular}




$\begin{array}{lcccc}\text { Estructura legislativa } & 3 & 2 & 9 & 14 \\ & (0.7) & (0.5) & (2.1) & (3.3) \\ \begin{array}{l}\text { Causas que señalan desprecio o } \\ \text { negación de la violencia contra las }\end{array} & 1 & 1 & 5 & 7 \\ \text { mujeres } & (0.2) & (0.2) & (1.2) & (1.7) \\ \text { Características físicas del agresor } & 0 & 3 & 1 & 4 \\ & & (0.7) & (0.2) & (0.9) \\ \text { Ns/Nc } & 9 & 27 & 66 & \\ & (2.1) & (6.4) & (15.6) & \end{array}$

En la Tabla 2 se incluyen los motivos señalados por las personas integrantes de la muestra como causa de esta violencia.

Llama especialmente la atención que, tanto los motivos considerados como principales en primer, segundo y tercer lugar, como las causas consideradas globalmente como más importantes, sean variables relacionadas con las características individuales de las personas que cometen la violencia (sentimientos de superioridad, celos / envidia, problemas de pareja, alcohol / drogas, etc.). Cabe señalar que, aunque el machismo sea considerado como causa principal de la violencia en tercer lugar, y se sitúe en cuarto lugar en el ranking global, únicamente es valorado como tal por un $10 \%$ y un $20 \%$ de las personas entrevistadas, respectivamente.

A continuación se procedió a realizar un análisis comparativo, diferenciando a las personas integrantes de la muestra en función de su sexo, edad, o nivel de estudios con objeto de analizar la posible relación entre estas variables y las creencias sobre la violencia de género. Dado el objetivo propuesto, se agruparon los resultados hasta diferenciar, en cada caso, categorías dicotómicas: a) considerar que la violencia de género es cometida por hombres o por otros colectivos; b) considerar que la violencia de género es padecida por mujeres o por otros colectivos; c) considerar que la violencia de género es frecuente a cualquier edad o en algún momento específico de la vida; d) considerar o no que la violencia de género se exagera; y e) considerar o no que las denuncias falsas en violencia de género son frecuentes. Los análisis por sexo (Tabla 3) muestran que no hay diferencias estadísticamente significativas entre hombres y mujeres en cuanto a sus creencias sobre quienes cometen o quienes padecen violencia de género, ni en cuanto a la posible exageración en relación con esta violencia. Sin embargo, el sexo sí está estadísticamente relacionado con las creencias sobre la edad a la que esta violencia es más frecuente, y sobre las denuncias falsas. Así, entre las mujeres es significativamente más elevada la proporción de quienes consideran que no hay edad para la ocurrencia de la violencia de género (45.4\%), mientras, entre los varones es significativamente superior la proporción de quienes creen que esta violencia es más frecuente en la adultez (41.8\%). Igualmente la proporción de varones que considera que se ponen muchas denuncias falsas en violencia de género es significativamente superior a la de mujeres que sostienen esta creencia.

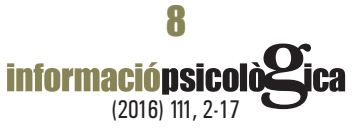


Tabla 3.- Creencias sobre la violencia de género. Comparación por sexo.

\begin{tabular}{|c|c|c|c|c|}
\hline & & $\begin{array}{c}\text { Hombres } \\
\mathrm{n}(\%)\end{array}$ & $\begin{array}{c}\text { Mujeres } \\
\mathrm{n}(\%)\end{array}$ & Significación \\
\hline \multirow{4}{*}{$\begin{array}{l}\text { Quiénes comenten } \\
\text { esta violencia }\end{array}$} & Hombres $(*)$ & $57(53.3)$ & $160(54.8)$ & $\chi^{2}(1)=.073$ \\
\hline & RTC & -.3 & .3 & $\mathrm{p}=.787$ \\
\hline & Otras respuestas & $50(46.7)$ & $132(45.2)$ & \\
\hline & RTC & .3 & -.3 & \\
\hline \multirow{4}{*}{$\begin{array}{l}\text { Quiénes son } \\
\text { víctimas } \\
\text { de esta violencia }\end{array}$} & Mujeres $(* *)$ & $53(49.1)$ & $149(49.7)$ & $\chi^{2}(1)=.011$ \\
\hline & RTC & -.1 & .1 & $\mathrm{p}=.916$ \\
\hline & Otras respuestas & $55(50.9)$ & $151(50.3)$ & \\
\hline & RTC & .1 & -.1 & \\
\hline \multirow{8}{*}{$\begin{array}{l}\text { En qué edad crees } \\
\text { que es más frecuente } \\
\text { esta violencia }\end{array}$} & No hay edad & $27(24.5)$ & $133(45.4)$ & $\chi^{2}(3)=16.558$ \\
\hline & RTC & -3.8 & 3.8 & $\mathrm{p}=.001$ \\
\hline & Adolesc-Juventud & $13(11.8)$ & 35 (11.9) & $\mathrm{Cc}=.199$ \\
\hline & RTC & .0 & .0 & \\
\hline & Adultez & $46(41.8)$ & $88(30.0)$ & \\
\hline & RTC & 2.2 & -2.2 & \\
\hline & Juventud+Adultez & $24(21.8)$ & $37(12.6)$ & \\
\hline & RTC & 2.3 & -2.3 & \\
\hline \multirow{4}{*}{$\begin{array}{l}\text { Crees que se exagera } \\
\text { el problema }\end{array}$} & $\mathrm{Si}$ & $9(7.8)$ & $13(4.2)$ & $\chi^{2}(1)=2.208$ \\
\hline & RTC & 1.5 & -1.5 & $\mathrm{p}=.137$ \\
\hline & No & $106(92.2)$ & $295(95.8)$ & \\
\hline & RTC & -1.5 & 1.5 & \\
\hline \multirow{4}{*}{$\begin{array}{l}\text { Crees que se ponen } \\
\text { muchas denuncias } \\
\text { falsas }\end{array}$} & $\mathrm{Si}$ & $44(38.3)$ & $83(26.9)$ & $\chi^{2}(1)=5.101$ \\
\hline & RTC & 2.3 & -2.3 & $\mathrm{p}=.024$ \\
\hline & No & $71(61.7)$ & $225(73.1)$ & $\mathrm{Cc}=.109$ \\
\hline & RTC & -2.3 & 2.3 & \\
\hline
\end{tabular}

(*) Para la comparación se ha incluido en esta categoría a las personas que respondieron hombres + personas de ambos sexos, pero mayoritariamente hombres + hombres con determinadas características

(**) Para la comparación se ha incluido en esta categoría a las personas que respondieron mujeres + personas de ambos sexos, pero mayoritariamente mujeres + mujeres con determinadas características

Cc: Coeficiente de contingencia 
Los resultados al considerar la edad de los encuestados (Tabla 4) indican que no hay diferencias estadísticamente significativas entre los diferentes grupos de edad en cuanto a sus creencias sobre quienes cometen violencia de género, o sobre la extensión de las denuncias falsas. La edad, en cambio, sí está estadísticamente relacionada con las creencias sobre

Tabla 4.- Creencias sobre la violencia de género. Comparación por edad.

\begin{tabular}{|c|c|c|c|c|c|c|}
\hline & & $\begin{array}{c}\text { De } 17 \text { a } \\
23 \text { años } \\
\text { n (\%) }\end{array}$ & $\begin{array}{c}\text { De } 24 \text { a } \\
40 \text { años } \\
\text { n }(\%)\end{array}$ & $\begin{array}{c}\text { De } 41 \text { a } \\
60 \text { años } \\
\text { n (\%) }\end{array}$ & $\begin{array}{c}\text { De } 60 \text { a } \\
81 \text { años } \\
\text { n (\%) }\end{array}$ & Significación \\
\hline \multirow{4}{*}{$\begin{array}{l}\text { Quiénes } \\
\text { comenten } \\
\text { esta violencia }\end{array}$} & Hombres $(*)$ & $98(57.0)$ & $33(45.8)$ & $40(54.8)$ & 14 (63.6) & $\chi^{2}(3)=3.350$ \\
\hline & RTC & .9 & -1.7 & .0 & .9 & $\mathrm{p}=.341$ \\
\hline & Otras respuestas & $74(43.0)$ & $39(54.2)$ & $33(45.2)$ & $8(36.4)$ & \\
\hline & RTC & -.9 & 1.7 & .0 & -.9 & \\
\hline \multirow{4}{*}{$\begin{array}{l}\text { Quiénes son } \\
\text { víctimas } \\
\text { de esta violencia }\end{array}$} & Mujeres $(* *)$ & 98 (55.7) & $26(35.1)$ & $38(51.4)$ & $12(57.1)$ & $\chi^{2}(3)=9.271$ \\
\hline & RTC & 2.0 & -3.0 & .2 & .6 & $\mathrm{p}=.026$ \\
\hline & Otras respuestas & 78 (44.3) & $48(64.9)$ & $36(48.6)$ & $9(42.9)$ & $\mathrm{Cc}=.162$ \\
\hline & RTC & -2.0 & 3.0 & -.2 & -.6 & \\
\hline \multirow{8}{*}{$\begin{array}{l}\text { En qué edad } \\
\text { crees } \\
\text { que es más } \\
\text { frecuente } \\
\text { esta violencia }\end{array}$} & No hay edad & $33(19.4)$ & $45(58.4)$ & $52(69.3)$ & $12(57.1)$ & $\chi^{2}(3)=74.525$ \\
\hline & RTC & -8.2 & 3.4 & 5.6 & 1.5 & $\mathrm{p}=.000$ \\
\hline & Adolesc-Juventud & $22(12.9)$ & $6(7.8)$ & $7(9.3)$ & $4(19.0)$ & $\mathrm{Cc}=.422$ \\
\hline & RTC & .9 & -1.1 & -.6 & 1.1 & \\
\hline & Adultez & 79 (46.5) & $18(23.4)$ & $10(13.3)$ & $3(14.3)$ & \\
\hline & RTC & 5.7 & -1.9 & -3.9 & -1.8 & \\
\hline & Juventud+Adultez & $36(21.2)$ & $8(10.4)$ & $6(8.0)$ & $2(9.5)$ & \\
\hline & RTC & 3.1 & -1.3 & -2.0 & -.7 & \\
\hline \multirow{4}{*}{$\begin{array}{l}\text { Crees que se } \\
\text { exagera } \\
\text { el problema }\end{array}$} & $\mathrm{Si}$ & $5(2.8)$ & $9(11.5)$ & $5(6.3)$ & $0(-)$ & $\chi^{2}(3)=9.741$ \\
\hline & RTC & -2.1 & 2.8 & .5 & -1.1 & $\mathrm{p}=.021$ \\
\hline & No & $175(97.2)$ & $69(88.5)$ & $74(93.7)$ & $22(100)$ & $\mathrm{Cc}=.163$ \\
\hline & RTC & 2.1 & -2.8 & -.5 & 1.1 & \\
\hline \multirow{4}{*}{$\begin{array}{l}\text { Crees que se } \\
\text { ponen } \\
\text { muchas } \\
\text { denuncias } \\
\text { falsas }\end{array}$} & $\mathrm{Si}$ & $58(32.2)$ & $22(28.2)$ & $20(25.3)$ & $3(13.6)$ & $\chi^{2}(3)=3.983$ \\
\hline & RTC & 1.5 & -.1 & -.8 & -1.6 & $p=.263$ \\
\hline & & $122(67.8)$ & $56(71.8)$ & $59(74.7)$ & $19(86.4)$ & \\
\hline & $\begin{array}{l}\text { No } \\
\text { RTC }\end{array}$ & -1.5 & .1 & .8 & 1.6 & \\
\hline
\end{tabular}


Tabla 5.- Creencias sobre la violencia de género. Comparación por nivel de estudios.

\begin{tabular}{|c|c|c|c|c|c|c|}
\hline & & $\begin{array}{l}\text { Prima- } \\
\text { rios } \\
n(\%)\end{array}$ & $\begin{array}{l}\text { Secun- } \\
\text { darios } \\
\mathrm{n}(\%)\end{array}$ & $\begin{array}{c}\text { Univer- } \\
\text { sitarios } \\
\mathrm{n}(\%)\end{array}$ & $\begin{array}{c}\text { Estu- } \\
\text { diantes Un. } \\
\mathrm{n}(\%)\end{array}$ & Signifi-cación \\
\hline \multirow{4}{*}{$\begin{array}{l}\text { Quiénes comenten } \\
\text { esta violencia }\end{array}$} & Hombres $(*)$ & $21(55.3)$ & $51(60.0)$ & $11(32.4)$ & $130(55.3)$ & $\chi^{2}(3)=7.826$ \\
\hline & RTC & .1 & 1.2 & -2.7 & .5 & $\mathrm{p}=.050$ \\
\hline & Otras respuestas & $17(44.7)$ & $34(40.0)$ & $23(67.6)$ & $105(44.7)$ & $\mathrm{Cc}=.140$ \\
\hline & RTC & -.1 & -1.2 & 2.7 & -.5 & \\
\hline \multirow{4}{*}{$\begin{array}{l}\text { Quiénes son } \\
\text { víctimas } \\
\text { de esta violencia }\end{array}$} & Mujeres $(* *)$ & $20(52.6)$ & $50(56.8)$ & $9(26.5)$ & $120(49.8)$ & $\chi^{2}(3)=9.253$ \\
\hline & RTC & .4 & 1.5 & -2.8 & .1 & $\mathrm{p}=.026$ \\
\hline & Otras respuestas & $18(47.4)$ & $38(43.2)$ & $25(73.5)$ & $121(50.2)$ & $\mathrm{Cc}=.150$ \\
\hline & RTC & -.4 & -1.5 & 2.8 & -.1 & \\
\hline \multirow{8}{*}{$\begin{array}{l}\text { En qué edad crees } \\
\text { que es más } \\
\text { frecuente } \\
\text { esta violencia }\end{array}$} & No hay edad & $21(63.6)$ & $58(65.2)$ & $20(52.6)$ & $59(24.9)$ & $\chi^{2}(3)=61.525$ \\
\hline & RTC & 2.9 & 5.6 & 1.7 & -7.4 & $\mathrm{p}=.000$ \\
\hline & Adolesc-Juventud & $4(12.1)$ & $6(6.7)$ & $3(7.9)$ & $33(13.9)$ & $\mathrm{Cc}=.366$ \\
\hline & RTC & .1 & -1.6 & -.7 & 1.8 & \\
\hline & Adultez & $6(18.2)$ & $15(16.9)$ & $7(18.4)$ & $104(43.9)$ & \\
\hline & RTC & -1.9 & -3.7 & -2.0 & 5.5 & \\
\hline & Juventud + Adultez & $2(6.1)$ & $10(11.2)$ & $8(21.1)$ & $41(17.3)$ & \\
\hline & RTC & -1.5 & -1.2 & 1.0 & 1.3 & \\
\hline \multirow{4}{*}{$\begin{array}{l}\text { Crees que se } \\
\text { exagera } \\
\text { el problema }\end{array}$} & $\mathrm{Si}$ & $4(10.3)$ & $3(3.3)$ & $1(2.6)$ & $14(5.6)$ & $\chi^{2}(3)=3.241$ \\
\hline & RTC & 1.5 & -1.0 & -.8 & .4 & $\mathrm{p}=.356$ \\
\hline & No & $35(89.7)$ & $88(96.7)$ & $37(97.4)$ & $234(94.4)$ & \\
\hline & RTC & -1.5 & 1.0 & .8 & -.4 & \\
\hline \multirow{4}{*}{$\begin{array}{l}\text { Crees que se ponen } \\
\text { muchas denuncias } \\
\text { falsas }\end{array}$} & $\mathrm{Si}$ & $10(25.6)$ & $22(24.2)$ & $11(28.9)$ & $83(33.5)$ & $\chi^{2}(3)=3.229$ \\
\hline & RTC & -.7 & -1.4 & -.2 & 1.7 & $\mathrm{p}=.358$ \\
\hline & No & $29(74.4)$ & $69(75.8)$ & $27(71.1)$ & $165(66.5)$ & \\
\hline & RTC & .7 & 1.4 & .2 & -1.7 & \\
\hline
\end{tabular}

quienes padecen esta violencia, sobre la edad de aparición, y sobre la posible exageración en relación con esta violencia. Así, entre las personas de 17 a 23 años hay una proporción significativamente superior de personas que consideran que las mujeres son las principales víctimas de la violencia de género (55.7\%), que ésta es más frecuente en la vida adulta (46.5\%), y que no se exagera en relación con esta violencia (97.2\%). Entre las personas de 24 a 60 años hay una proporción significativamente superior de personas que consideran 
que no hay una edad específica para la ocurrencia de esta violencia $(58.4 \%$ y $69.3 \%$, respectivamente). Y, entre las personas de 24 a 40 años hay una proporción significativamente más elevada de personas que no consideran a las mujeres como principales víctimas de la violencia de género (64.9\%) y que ésta se exagera (11.5\%).

Finalmente, los resultados obtenidos al diferenciar a las personas integrantes de la muestra en función de su nivel de estudios (Tabla 5) indican que no hay relación entre ésta variable y las creencias sobre una posible exageración de la violencia de género, o sobre la extensión de las denuncias falsas. El nivel de estudios parece estar asociado significativamente con las creencias sobre quienes cometen y padecen esta violencia y sobre la edad a la que esta violencia es más frecuente. Así, la proporción de personas con estudios universitarios que no considera a los varones como principales perpetradores de violencia de género (67.6\%) ni a las mujeres como sus principales víctimas (73.5\%) es significativamente superior al resto; las personas con estudios primarios o secundarios, por su parte, consideran, en una proporción significativamente superior al resto, que no hay una edad específica en que la violencia de género sea más prevalente (63.6\% y $65.2 \%$, respectivamente); y el alumnado universitario considera, en una proporción significativamente superior al resto, esta violencia como más frecuente en la vida adulta (43.9\%).

\section{Discusión}

En términos generales, los resultados obtenidos muestran que los mitos sobre la violencia de género, tanto en cuanto a quien la comete, como a quien la padece, a sus causas, al momento vital en que ocurre o a la existencia de denuncias falsas, tienen aún vigencia en nuestro entorno.

Sobre quienes padecen o cometen esta violencia y sus características, evidencias anteriores, tanto a nivel internacional como nacional, procedentes de numerosas encuestas (DGVG, 2015; FRA, 2014; García-Moreno, 2005; OMS, 2013; OPS, 2013) y análisis sobre el tema (Fortes, 2012; Lorente, 2001, 2009), concluye que esta violencia es cometida en una abrumadora mayoría por los varones y es padecida por las mujeres y sus hijos e hijas, y que no existiría un perfil determinado o característico de varón maltratador ni de mujer maltratada. No en vano, en el propio ordenamiento jurídico español se explicita que violencia de género es aquella cometida por varones, y padecida por mujeres con las que éstos tenían, o habían tenido un vínculo sentimental, exclusivamente.

Sin embargo, los resultados obtenidos en este trabajo, al igual que otros previos, realizados tanto con población general (Comisión Europea, 1999, 2010; González et al., 2011), como con alumnado universitario (Ferrer, Bosch, Ramis, Torrens y Navarro, 2006), y con profesionales sanitarios (Arredondo, Broco, Alcalá, Rivera, Jiménez y Gallardo, 2012), han detectado la persistencia de creencias erróneas, estereotipos y mitos en relación con estas cuestiones en una proporción no desdeñable (en torno a un tercio) de personas encuestadas, y tanto en general, como entre hombres y mujeres, y personas de diferentes edades, niveles de estudios o cualificaciones profesionales.

Por lo que se refiere a las causas de la violencia de género, tomando en consideración el carácter complejo de esta violencia (Heise, 1997), actualmente hay coincidencia en considerar 
que su análisis debería ser realizado desde modelos explicativos de tipo multicausal (ONU, 2006; OPS, 2013). La característica común a estos modelos es que, aunque puedan diferir en la importancia que asignan a los diferentes factores individuales y sociales considerados, todos ellos entienden esta violencia como un fenómeno complejo, que sólo puede ser explicado a partir de la intervención de un conjunto de factores específicos, en el contexto general de las desigualdades de poder entre varones y mujeres, en los niveles individual, grupal, nacional y mundial (ONU, 2006). Es decir, estos modelos no sólo toman en consideración la existencia de múltiples factores que contribuyen a la génesis y/o mantenimiento de esta violencia, sino que entienden que el género y las relaciones de género desempeñan un papel clave en la violencia ejercida por los hombres hacia las mujeres (APA, 1999), y vinculan esta violencia con una sociedad dicotomizada y estructurada desigualmente en función del género (Delgado, 2013), si bien la importancia que otorgan a estos factores puede variar de unos modelos a otros.

A pesar de ello, tanto los datos procedentes de los Eurobarómetros (Comisión Europea, 1999, 2010), como de estudios realizados con población española general (CIS, 2005), o universitaria (Ferrer, Bosch, Ramis y Navarro, 2005), muestran que el análisis social de las causas de la violencia de género se aleja de estas evidencias, y sitúa como principales motivos para su ocurrencia las causas individuales, incluyendo el abuso de alcohol u otras sustancias, o la enfermedad mental, el paro, la pobreza, etc. Al igual que ya sucedía en esos estudios previos, ciertos factores estructurales relacionados con el sistema patriarcal imperante (como el reparto de poder entre hombres y mujeres, el sexismo, o la desigualdad) aparecen como causas emergentes, valoradas por sectores más o menos importantes de población como causas de la violencia de género, pero sin llegar a ser percibidos como causas centrales o nucleares de la misma. Los resultados obtenidos en este trabajo van en esta misma líneas, mostrando que la población encuestada centra su análisis sobre las causas de esta violencia en razones de tipo individual.

Otro resultado de interés es que, aunque la proporción de quienes perciben que se exagera en torno al problema de la violencia de género se ha reducido (Comisión Europea, 1999, 2010; Ferrer et al., 2005), los resultados obtenidos en este trabajo muestran que se mantiene el mito de las denuncias falsas. En relación con esta cuestión, cabe recordar que, en determinados medios, tanto de divulgación como científicos (i.e., Pérez y Bernabé, 2012), se viene insistiendo en la existencia de denuncias falsas interpuestas por mujeres que no han padecido violencia de género y que, aprovechándose de la legislación vigente, intentarían vengarse de su pareja o ex - pareja, y/o obtener beneficios ante una separación o divorcio. Tal y como ha quedado reflejado en los resultados presentados, esta creencia está extendida entre un porcentaje no desdeñable de la población encuestada (en torno a un tercio, y, particularmente, entre los varones). Sin embargo, los estudios sobre el tema desmienten reiteradamente que el número de estas denuncias sea significativo (Bosch y Ferrer, 2012; Cabruja, 2009; Del Pozo, 2013; Larrauri, 2008). Los documentos de organismos especializados, como la Memoria de la Fiscalía General del Estado (2014), que analizó los procedimientos por denuncias falsas desde 2009 a 2013, lo corroboran concluyendo que: 
El escasísimo porcentaje de denuncias incoadas cada año por delito de acusación y denuncia falsa - 120 - en relación al número de denuncias por violencia contra la mujer interpuestas - 657.084 - que supone un 0,018\% es suficientemente elocuente para rebatir las voces que se alzan en torno a la "prevalencia de denuncias falsas" en materia de violencia contra las mujeres (p. 311).

En relación con esta cuestión, cabe, además, señalar que en algunos casos se ha detectado la existencia de contradenuncias (Amnistía Internacional, 2012; Ortubay, 2015), esto es, de denuncias interpuestas por los agresores hacia las víctimas en base a hechos como, por ejemplo, las posibles lesiones causadas por éstas mientras se estaban defendiendo de una agresión. La sospecha de la existencia de este tipo de prácticas requiere un análisis en profundidad, y, en su caso, arbitrar los mecanismos para que, garantizando adecuadamente los derechos y posibilidades de todas las personas intervinientes en el proceso, estas prácticas no lleguen a constituir una barrera cuando la mujer víctima se plantea interponer una denuncia por violencia de género.

Respecto a las limitaciones del estudio, cabe señalar en primer lugar que, la muestra estudiada no es representativa de la población, debido tanto a su limitado tamaño, como al uso de un criterio de muestreo no probabilítico, como a su composición (mayor proporción de alumnado universitario y mujeres). Por ello los resultados obtenidos en este trabajo no son generalizables a la población general. En segundo lugar, el propio cuestionario empleado constituye una limitación puesto que no está validado y recoge, principalmente, información de carácter cualitativo. Sin embargo, y a pesar de ello, estos resultados se consideran de interés a efectos exploratorios ya que permiten avanzar la vigencia actual de los mitos estudiados en segmentos importantes de la población, y también hipotetizar que éstos se hallan ampliamente extendidos entre personas con diferentes características y, entre mujeres y hombres y personas de diferentes edades y niveles de estudios.

En este sentido, los resultados obtenidos constituyen una evidencia que se suma a otras previamente disponibles, y que remarca la necesidad de seguir trabajando tanto para deshacer los mitos socialmente admitidos en relación con la violencia de género, como para incrementar la sensibilización social, especialmente, la de aquellas personas que, en razón de su formación o práctica profesional puedan llegar a tener un contacto directo con esta violencia. De hecho, se considera que ésta es la principal y más importante aportación y significación práctica de este trabajo: la constatación de que es imprescindible continuar desarrollando intervenciones para la formación específica y la sensibilización social en torno a la violencia de género, que permitan alcanzar un conocimiento ajustado al respecto y desmontar los mitos aún vigentes. A la luz de los resultados obtenidos, cabe insistir en la importancia de destinar estas intervenciones a personas de toda edad y condición como también a colectivos específicos (profesionales, con estudios o sin ellos, de toda edad, etc.) ya que, como queda de manifiesto en dichos resultados, ni la edad, ni el nivel de estudios protegen o liberan a las personas de estas creencias erróneas. 
Igualmente, y como propuestas futuras de investigación, cabe seguir indagando en la persistencia de estos mitos en muestras más amplias y en otros segmentos poblacionales, así como indagar sobre otras variables relacionadasy también sobre otras formas de violencia contra las mujeres, como las agresiones sexuales (Romero y López-Mejías, 2009; Sagrillo, Martíns y Nogueira, 2014; Saldívar, Ramos y Saltijeral, 2004; Suárez y Gadalla, 2010; Watherhouse, Reynolds y Egan, 2016), para establecer posibles mecanismos y claves comunes que permitan, posteriormente, trabajar de modo conjunto en el desmontaje de todos ellos, y así optimizar al máximo las intervenciones que puedan planificarse.

\section{Agradecimientos}

Las autoras de este trabajo queremos agradecer la colaboración prestada por la Sra. Lourdes Barros Garrido y la Dra. Joanna Blahopoulou en el reclutamiento de integrantes de la muestra, recogida de cuestionarios, y tareas de categorización de las respuestas obtenidas.

\section{Referencias}

Amnistía Internacional (2012). ¿Qué justicia especializada? A siete años de la Ley Integral contra la Violencia de Género: obstáculos al acceso y obtención de justicia y protección. Madrid: Autor.

APA (American Psychological Association) (1999).Resolution On Male Violence Against Women. Recuperado de: http://www.apa.org/about/policy/male-violence.aspx

Arredondo, A. B., Broco, M., Alcalá, T., Rivera, A., Jiménez, M. I. y Gallardo, C. (2012). Profesionales de atención primaria de Madrid y violencia de pareja hacia la mujer en el año 2010. Revista Española de Salud Pública, 86(1), 85-99.

Bosch, E. y Ferrer, V. A. (2002). Las víctimas de un mal amor que mata. Madrid: Cátedra. Colección Feminismos.

Bosch, E. y Ferrer, V. A. (2012). Nuevo mapa de los mitos sobre la violencia de género en el s. XXI. Psicothema, 24(4), 548-554.

Cabruja, T. (2009). 'Testimoni@s/activ@smolest@s.' Prácticas discursivas y dispositivos sociosexuados en psicología y derecho. En G. Nicolás y E. Bodelón (Comps.), Género y dominación: críticas feministas del derecho y el poder (pp. 127-158). Barcelona: Anthropos.

Centro de Investigaciones Sociológicas (CIS) (2005). Avance de resultados. Barómetro de marzo de 2005. Estudio 2597. Recuperado de: http://www.cis.es/baros/mar2597.htm

Comisión Europea (1999). L'opinion des europeens sur la violence domestique dont sont victimes les femmes. Recuperado de: http://europa.eu.int/comm/dg10/epo/eb/eb51/violence fr.pdf

Comisión Europea (2010). Domestic violence against women. Special Eurobarometer 73.2. Belgium: TNS Opinión \& Social. Recuperado de: http://ec.europa.eu/public opinion/archives/ebs/ebs 344 en.pdf

Del Pozo, M. (2013). Rompiendo el mito de las denuncias falsas en violencia de género. En A. Figueruela. M. del Pozo y M. León (Dirs.), Violencia de género e igualdad: una cuestión de derechos humanos (pp. 55-64). Granada: Comares.

Delgado, C. (2013). Aspectos cognitivos y emocionales de la violencia de género. En A. Figueruela. M. del Pozo y M. León (Dirs.), Violencia de género e igualdad: una cuestión de derechos humanos (pp. 65-79). Granada: Comares.

DGVG (Delegación del Gobierno para la Violencia de Género) (2015). Macroencuesta de violencia contra la mujer 2015. Avance de resultados. Madrid: Ministerio de Sanidad, Servicios Sociales e Igualdad. Recuperado de: http://www.msssi.gob.es/ssi/violenciaGenero/publicaciones/estudiosinvestigaciones/PDFS/ AVANCE MACROENCUESTA DE VIOLENCIA CONTRA LA MUJER 2015.pdf 
Ferrer, V. A., Bosch, E., Ramis, M. C. y Navarro, C. (2005). La percepción de la violencia contra las mujeres en la pareja en estudiantes universitarios/as. Revista de Psicología Social Aplicada, 15(3), 61-82.

Ferrer, V. A., Bosch, E., Ramis, M. C., Torrens, G. y Navarro, C. (2006). La violencia contra las mujeres en la pareja: creencias y actitudes en estudiantes universitarios/as. Psicothema, 18(3), 359-366.

Fiscalía General del Estado (2014). Memoria de la Fiscalía General del Estado 2014. Recuperado de: https:// www.fiscal.es/fiscal/publico/ciudadano/documentos/memorias fiscalia general estado/!ut/p/ a1/04 Sj9CPykssy0xPLMnMzOvMAfGjzOI9HTOcDT2DDbwsgozNDBwtjNycnDx8jAwszIAKIpEVuPtYuBk4unsG05I6eBhbBJkQp98AB3A0IKQ XD8KVYm h68R0AWGvqEmli7GBu6G6AqwOBGsAI8bCnJDIwwyPRUByaZ9Ig!!/dI5/d5/L2dBISEvZOFBIS9nQSEh/?selAnio = 2014

Fortes, D. (2012). Características de los hombres que maltratan a sus parejas. Clepsydra: revista de estudios de género y teoría feminista, 11,121-132.

FRA (European Union Agency for Fundamental Rights) (2014).Violence against women: an EU-wide Surrey. Main results. Luxembourg: Publications Office of the European Union. Recuperado de: http://fra.europa. eu/sites/default/files/fra-2014-vaw-survey-main-results en.pdf

García Moreno, C. (2005) (Coord.). Estudio multipaís de la OMS sobre salud de la mujer y violencia doméstica. Primeros resultados sobre prevalencia, eventos relativos a la salud y respuestas de las mujeres a dicha violencia. Ginebra: Organización Mundial de la Salud. Recuperado de http://whqlibdoc. who.int/publications/2005/924359351X spa.pdf

González, I., Serrano, A., García, N., Giménez, M. del C., Moreno, B., González, A. B., Montoya, J., Téllez, J. M. y Escobar, F. (2011). Conocimientos sobre violencia de género de la población que consulta en Atención Primaria. Atención Primaria, 43(9), 459-464.

Heise, L. L. (1997). La violencia contra la mujer. Organización global para el cambio. En Jeffrey L. Edleson, y Zvi C. Eisikovits (Eds.), Violencia doméstica: La mujer golpeada y la familia (pp. 19-58). Barcelona: Granica.

Larrauri, E. (2008). Cinco tópicos sobre las mujeres víctimas de violencia. En P. Laurenzo, M.L. Maqueda y A. Rubio (Coord.), Género, violencia y derecho (pp. 311-327). Valencia: Tirant lo Blanch.

Lorente, M. (2001). Mi marido me pega lo norma.I Agresión a la mujer: realidades y mitos. Barcelona: Ares y Mares.

Lorente, M. (2009). El maltratador, la condición masculina y el maltrato a las mujeres. Crítica, 59(960), 44-47.

Lorente, M. (2009). Los nuevos hombres nuevos. Barcelona: Destino.

Méndez, R. (2010). El SAP en su repercusión social. En A. Escudero, D. González, R. Méndez, C. Naredo, E. Pleguezuelos y S. Vaccaro (Eds.), Informe del Grupo de Trabajo de Investigación sobre el Supuesto Síndrome de Alienación Parental (pp. 76-94). Madrid: Ministerio de Igualdad.

OMS (Organización Mundial de la Salud) (2013). Estimaciones mundiales y regionales de la violencia contra la mujer: prevalencia y efectos de la violencia conyugal y de la violencia sexual no conyugal en la salud. Ginebra, Suiza: Autor. Recuperado de: http://www.who.int/reproductivehealth/publications/ violence/9789241564625/es/index.html

ONU (Organización de Naciones Unidas) (2006). Estudio a fondo sobre todas las formas de violencia contra la mujer (AG 61/122/Add.1). Nueva York: Autor. Recuperado de: http://www.observatorioviolencia.org/ documentos.php?id=92

OPS (Organización Panamericana de la Salud) (2013b).Violencia infligida por la pareja. Serie: Comprender y abordar la violencia contra las mujeres. Washington: Organización Mundial de la Salud.

Ortubay, M. (2015). Cuando la Respuesta Penal a la Violencia Sexista se Vuelve contra las Mujeres: las Contradenuncias. Oñati. Socio-legal Series, 5(2), 645-668.

Pérez, F. y Bernabé, B. (2012). Las denuncias falsas en casos de violencia de género ¿Mito o realidad? Anuario de PsicologíaJurídica, 22, 37-46.

Peters, J. (2008). Measuring myths about domestic violence: development and initial validation of the domestic violence myth acceptance scale. Journal of Aggression, Maltreatment \& Trauma, 16(1), 1-21. 
Romero, M. y López-Mejías, J. (2009). Agresiones sexuales en población universitaria: El papel del alcohol y de los mitos sobre la violación. International Journal of Psychological Research, 2(1), 44-53.

Sagrillo, A., Martins, V. y Nogueira, C. (2014). Adaptação da Escala de Aceitação dos Mitos de Estupro: evidências de validade. AvaliaçaoPsicologica: Interamerican Journal of Psychological Assessment, 13(1), 57-65.

Saldívar, G., Ramos, L. y Saltijeral, M. T. (2004). Validación de las escalas de aceptación de la violencia y de los mitos de violación en estudiantes universitarios. Salud Mental, 27(6), 40-49.

San Martín, C. y González, A. (2011). Las mujeres víctimas de violencia en los discursos psicológicos: ¿Espejos deformantes? Estudios de Psicología, 32(3), 405-417.

Suárez, E. y Gadalla, T. M. (2010). Stop blaming the victim:a meta - analysis of rape myths. Journal of Interpersonal Violence, 25(11), 2010-2035.

Waterhouse, G. F., Reynolds, A. yEgan, V. (2016). Myths and legends: the reality of rape ofeences reported to a UK. The European Journal of Psychology Applied to Legal Context, 8(1), 1-10. 